\title{
Analysis of subbrow upper blepharoplasty by measuring the lid-to-brow distance
}

\author{
Woo Ju Kim, Han Koo Kim, \\ Tae Hui Bae, Woo Seob Kim \\ Department of Plastic and Reconstructive \\ Surgery, Chung-Ang University Hospital, \\ Chung-Ang University College of \\ Medicine, Seoul, Korea
}

This article was presented at the 2018 Plastic Surgery The Meeting Resident Scientific Session on September 28 to October 1, 2018, in Chicago, IL, USA.
Background Conventional upper blepharoplasty through a supratarsal incision is commonly performed for periorbital rejuvenation. However, the drawbacks of this technique include insufficient removal of lateral hooding and an unnatural postoperative appearance. Subbrow upper blepharoplasty with submuscular fascia fixation was developed to avoid these shortcomings. Herein, we report our experiences with this technique and present an objective analysis of the results.

Methods Subbrow upper blepharoplasty was performed in 30 patients between June 2016 and October 2017. All patients had preoperative frontal view photographs, and the distance from the upper lid margin to the lower edge of the eyebrow was measured at the lateral limbus (LBD-L).

Results Between preoperative and postoperative measurements, the LBD-L was shortened by an average of 0.800 and $0.833 \mathrm{~mm}$ on the right and left, respectively $(P=0.047$ and $\mathrm{P}=0.070$ ). Most patients were satisfied with their aesthetic appearance and the improvement of their visual field.

Conclusions The lid-to-brow distance was minimally changed by the procedure, but the results were aesthetically favorable. This seemingly paradoxical finding is explained by the characteristics of the surgical procedure and the related anatomy. Among the various possible blepharoplasty techniques, subbrow upper blepharoplasty with submuscular fascia fixation has definite value for Asians.

Keywords Eyelids / Blepharoplasty / Eyebrow

\section{INTRODUCTION}

As Korea has developed into an aging society with evolving socioeconomic standards, upper blepharoplasty has become one of the most popular rejuvenation procedures. Conventional upper blepharoplasty through a supratarsal incision has been widely accepted,

Received: Jan 18, 2019 Revised: Feb 17, 2019 Accepted: Feb 21, 2019 Correspondence: Woo Seob Kim Department of Plastic and Reconstructive Surgery, Chung-Ang University Hospital, 102 Heukseok-ro, Dongjak-gu, Seoul 06973, Korea

Tel: +82-2-6299-1627, Fax: +82-2-823-1049, E-mail: kimws@cau.ac.kr

Copyright @ 2019 The Korean Society for Aesthetic Plastic Surgery.

This is an Open Access article distributed under the terms of the Creative Commons Attribution Non-Commercial License (http://creativecommons.org/licenses/by-nc/4.0/) which permits unrestricted non-commercial use, distribution, and reproduction in any medium, provided the original work is properly cited. $\quad w w w . e-a a p s . o r g$ and involves excision of redundant skin and muscle with or without creation of an eyelid fold.

However, the drawbacks of this technique, including insufficient removal of redundant lateral skin, a scar beyond the lateral canthus, and an unnatural postoperative appearance, have led to plastic surgeons to avoid conventional upper blepharoplasty [1]. Moreover, since Asians tend to have higher eyebrows than Caucasians and more pretarsal and suborbicularis fat, the drawbacks of the conventional procedure have become increasingly apparent $[2,3]$.

The infrabrow excision blepharoplasty technique was used by many Korean surgeons even before it was first reported. This technique includes elliptical excision of the infrabrow skin and subcutaneous tissue with or without the orbicularis oculi muscle (OOM) to alleviate lid redundancy [4]. However, only a few reports have described suture fixation of the OOM in the flap inferior to the supraorbital rim [5]. 
Subbrow blepharoplasty (subbrow lift) with submuscular fascia fixation is an advanced infrabrow excision technique that addresses the shortcomings of conventional blepharoplasty and allows the patient's natural eyelid crease or previously created surgical lid crease to be defined more clearly, while correcting lateral hooding and avoiding an unnatural postoperative appearance.

Herein, we introduce subbrow blepharoplasty with submuscular fascia fixation to correct upper lid redundancy. Changes in periorbital adnexal distances were measured to confirm the usefulness of this upper blepharoplasty technique.

\section{METHODS}

\section{Patients}

Sixty upper lids in 30 patients underwent subbrow upper blepharoplasty between June 2016 and October 2017. The primary indications for the procedure included: (1) upper lid dermatochalasis with lateral hooding; (2) desire to maintain the natural lid crease; (3) prior lid operations; (4) consideration of eyebrow tattooing; and (5) desire for a more natural contour with rapid recovery.

All patients had sitting preoperative frontal view photographs of the eyes, and the distance from the upper lid margin to the lower edge of the eyebrow was measured at the lateral limbus (LBD-L) (Fig. 1). These measurements were repeated immediately after surgery and at every follow-up visit for 6 months.

The experimental design was approved by the Institutional Review Board of Chung-Ang University Hospital (IRB No. 1902-00116245 ) and performed in accordance with the principles of the Declaration of Helsinki.

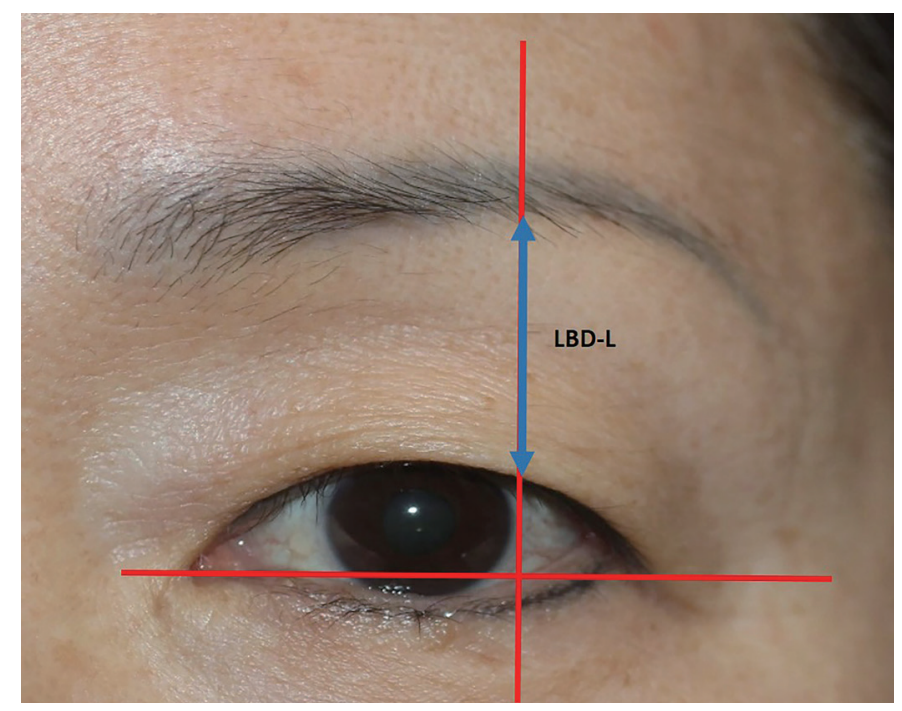

Fig. 1. The distance from upper lid margin to the lower edge of the eyebrow at lateral limbus (LBD-L) was measured before surgery and 6 months after surgery.

\section{Surgical techniques}

With the patient supine on the operating table, the location of the supraorbital nerve was marked by palpating the supraorbital notch. An upper incision line was drawn by following the inferior margin of the eyebrow, and the mid-pupil plane and brow peak point were marked over the eyebrow. The amount of skin to be excised was determined by pinching the surplus skin with forceps in the infrabrow area. A lower incision line was then drawn in a lazy-S or scal-

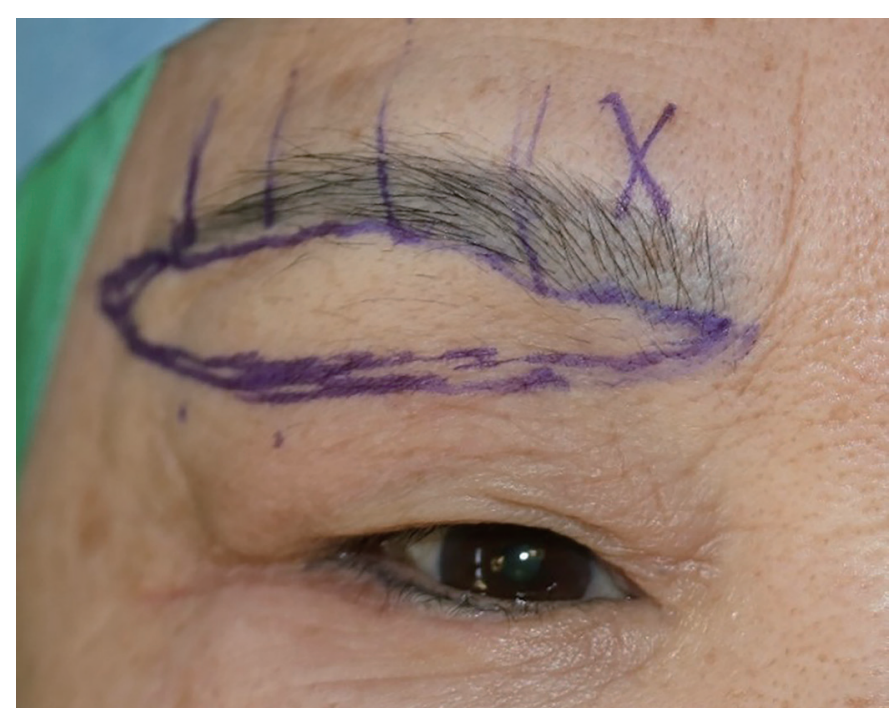

Fig. 2. An example of surgical design. The upper incision followed the lower border of the eyebrow, and the lower incision widened laterally in a scalpel or lazy-S shape. The mid-pupil point and brow peak point were marked for suture fixation.

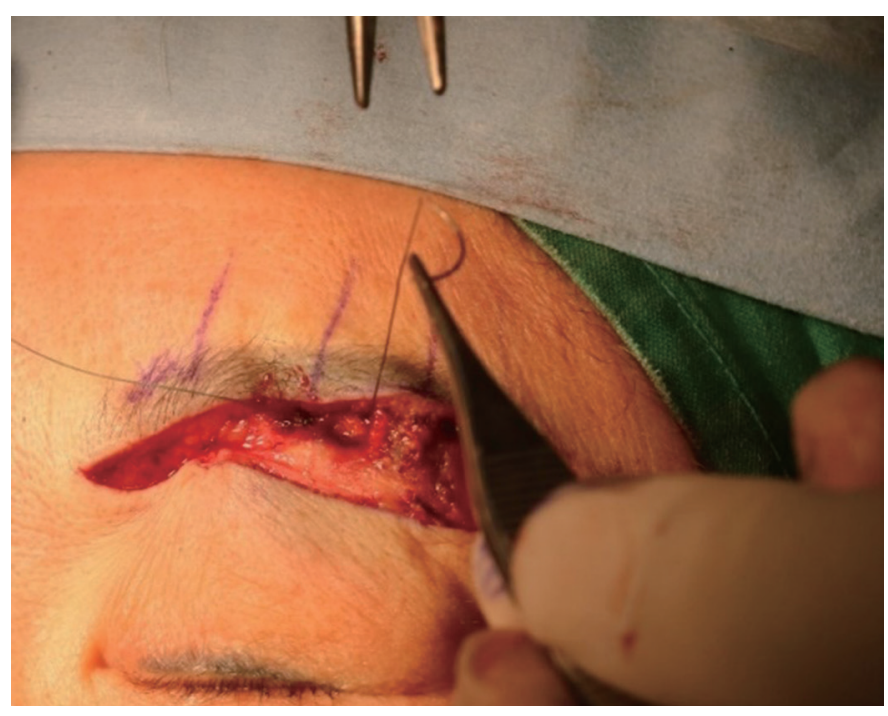

Fig. 3. Transverse 4-0 Ethibond sutures were fixed to the submuscular fascia, just above the supraorbital rim periosteum. 
pel shape, increasing in width laterally based on the amount of skin to be excised (Fig. 2).

Under local anesthesia using 2\% lidocaine with 1:100,000 epinephrine solution, incisions were made with a no. 15 blade. To avoid hair follicle injury, the upper incision was made in a beveled fashion, along with hair follicles. A lower incision was made deep to the level of the OOM. Starting from the most lateral end of the incision, skin and OOM excision proceeded medially and became more superficial at the medial end to avoid nerve injury.

To fix the OOM on the inferior flap, we made a slit incision over the supraorbital rim by splitting the muscle with small Metzenbaum scissors. The fixation points were usually at the mid-pupil plane, brow peak point, and $1 \mathrm{~cm}$ lateral to the brow peak point, as previ- ously marked during preoperative design. The number of fixation points can be altered based on an individual patient's eyebrow contour and the degree of lateral hooding. The anchoring depth of the OOM in the inferior flap was determined by the thickness of soft tissue in the upper lid. Three or more transverse 4-0 Ethibond sutures were fixed to the submuscular fascia, just above the supraorbital rim periosteum (Fig. 3).

Subcutaneous sutures were placed using 6-0 Monocryl and the skin was closed with 6-0 or 7-0 nylon.

\section{Statistical analysis}

Preoperative and postoperative LBD-L values were analyzed using SPSS version 19.0 (IBM Corp., Armonk, NY, USA) and differences

Table 1. Patient demographics

\begin{tabular}{|c|c|c|c|c|c|c|c|}
\hline No. & Sex/age (yr) & $\begin{array}{l}\text { Preoperative LBD-L } \\
\text { (right/left, } \mathrm{mm} \text { ) }\end{array}$ & $\begin{array}{l}\text { Postoperative LBD-L } \\
\text { (right/left, mm) }\end{array}$ & $\begin{array}{l}\text { Resection width } \\
\text { (mm] }\end{array}$ & $\begin{array}{l}\text { Eyelid } \\
\text { crease }\end{array}$ & $\begin{array}{l}\text { Follow-up period } \\
\text { (mon) }\end{array}$ & Complications \\
\hline 1 & $F / 58$ & $19 / 21$ & $19 / 20$ & 8 & $\mathrm{~S}$ & 6 & No \\
\hline 2 & $F / 66$ & $20 / 20$ & $20 / 20$ & 9 & $\mathrm{~S}$ & 7 & No \\
\hline 3 & $F / 69$ & $23 / 23$ & $23 / 22$ & 10 & $\mathrm{~S}$ & 12 & No \\
\hline 4 & $F / 58$ & $23 / 22$ & $22 / 21$ & 8 & $\mathrm{D}$ & 14 & No \\
\hline 5 & $\mathrm{~F} / 62$ & $19 / 20$ & $19 / 20$ & 10 & $\mathrm{~S}$ & 8 & No \\
\hline 6 & $F / 49$ & $18 / 19$ & $18 / 18$ & 11 & $D$ & 20 & No \\
\hline 7 & $F / 62$ & $25 / 26$ & $24 / 25$ & 10 & $\mathrm{~S}$ & 12 & No \\
\hline 8 & $\mathrm{~F} / 55$ & $23 / 21$ & $21 / 21$ & 10 & $\mathrm{D}$ & 6 & No \\
\hline 9 & $\mathrm{~F} / 57$ & $25 / 25$ & $24 / 24$ & 10 & $\mathrm{D}$ & 8 & No \\
\hline 10 & $F / 61$ & $26 / 24$ & $25 / 24$ & 10 & $\mathrm{D}$ & 10 & No \\
\hline 11 & $\mathrm{~F} / 75$ & $25 / 25$ & $23 / 24$ & 11 & $\mathrm{~S}$ & 27 & No \\
\hline 12 & $F / 29$ & $23 / 23$ & $23 / 23$ & 9 & $\mathrm{~S}$ & 21 & No \\
\hline 13 & $M / 75$ & $30 / 31$ & $29 / 26$ & 12 & $\mathrm{~S}$ & 6 & No \\
\hline 14 & $\mathrm{~F} / 78$ & $25 / 27$ & $25 / 26$ & 10 & $\mathrm{~S}$ & 6 & No \\
\hline 15 & $F / 62$ & $26 / 27$ & $26 / 26$ & 9 & $D$ & 9 & No \\
\hline 16 & $\mathrm{~F} / 59$ & $25 / 25$ & $22 / 22$ & 10 & $D$ & 15 & No \\
\hline 17 & $F / 53$ & $29 / 27$ & $26 / 26$ & 8 & $D$ & 20 & No \\
\hline 18 & $M / 56$ & $21 / 20$ & $29 / 29$ & 10 & $D$ & 6 & No \\
\hline 19 & $\mathrm{~F} / 53$ & $24 / 25$ & $23 / 24$ & 9 & $D$ & 8 & No \\
\hline 20 & $\mathrm{~F} / 75$ & $24 / 23$ & $24 / 23$ & 10 & $D$ & 9 & No \\
\hline 21 & $F / 61$ & $24 / 26$ & $23 / 25$ & 8 & $D$ & 14 & No \\
\hline 22 & $F / 43$ & $22 / 22$ & $21 / 21$ & 10 & $\mathrm{~S}$ & 23 & No \\
\hline 23 & $M / 65$ & $29 / 28$ & $25 / 23$ & 10 & $D$ & 6 & No \\
\hline 24 & $M / 60$ & $18 / 18$ & $18 / 18$ & 8 & $D$ & 9 & No \\
\hline 25 & $F / 62$ & $15 / 15$ & $15 / 14$ & 10 & $\mathrm{~S}$ & 6 & No \\
\hline 26 & $F / 63$ & $28 / 26$ & $23 / 22$ & 10 & $D$ & 12 & No \\
\hline 27 & $\mathrm{~F} / 55$ & $22 / 21$ & $20 / 23$ & 9 & $D$ & 14 & No \\
\hline 28 & $F / 59$ & $17 / 16$ & $16 / 16$ & 9 & $D$ & 6 & No \\
\hline 29 & $F / 65$ & $22 / 24$ & $20 / 20$ & 9 & $\mathrm{~S}$ & 9 & No \\
\hline 30 & $F / 58$ & $26 / 26$ & $26 / 25$ & 8 & D & 9 & No \\
\hline
\end{tabular}

LBD-L, the distance from upper lid margin to the lower edge of the eyebrow at lateral limbus; F, female; $S$, single fold; $D$, double fold; M, male. 
were considered to be statistically significant if $\mathrm{P}$-values were $<0.05$. The paired $t$-test was used to characterize the statistical significance of differences between preoperative and postoperative LBD-L measurements. Correlations between differences in LBD-L values and resection width or eyelid crease were analyzed using the Spearman correlation test and the Fisher exact test.

\section{RESULTS}

Thirty patients (60 eyelids) underwent subbrow upper blepharoplasty between June 2016 and October 2017. The average age of

Table 2. Preoperative and postoperative lid-to-brow distance at the lateral limbus

\begin{tabular}{lcccc}
\hline & Preoperative & Postoperative & $\begin{array}{c}\text { Average } \\
\text { difference }\end{array}$ & P-value \\
\hline LBD-L (right) & 23.20 & 22.40 & -0.800 & 0.047 \\
LBD-L (left) & 23.19 & 22.37 & -0.833 & 0.070 \\
\hline
\end{tabular}

The unit of value is millimeters.

LBD-L, the distance from upper lid margin to the lower edge of the eyebrow at lateral limbus.
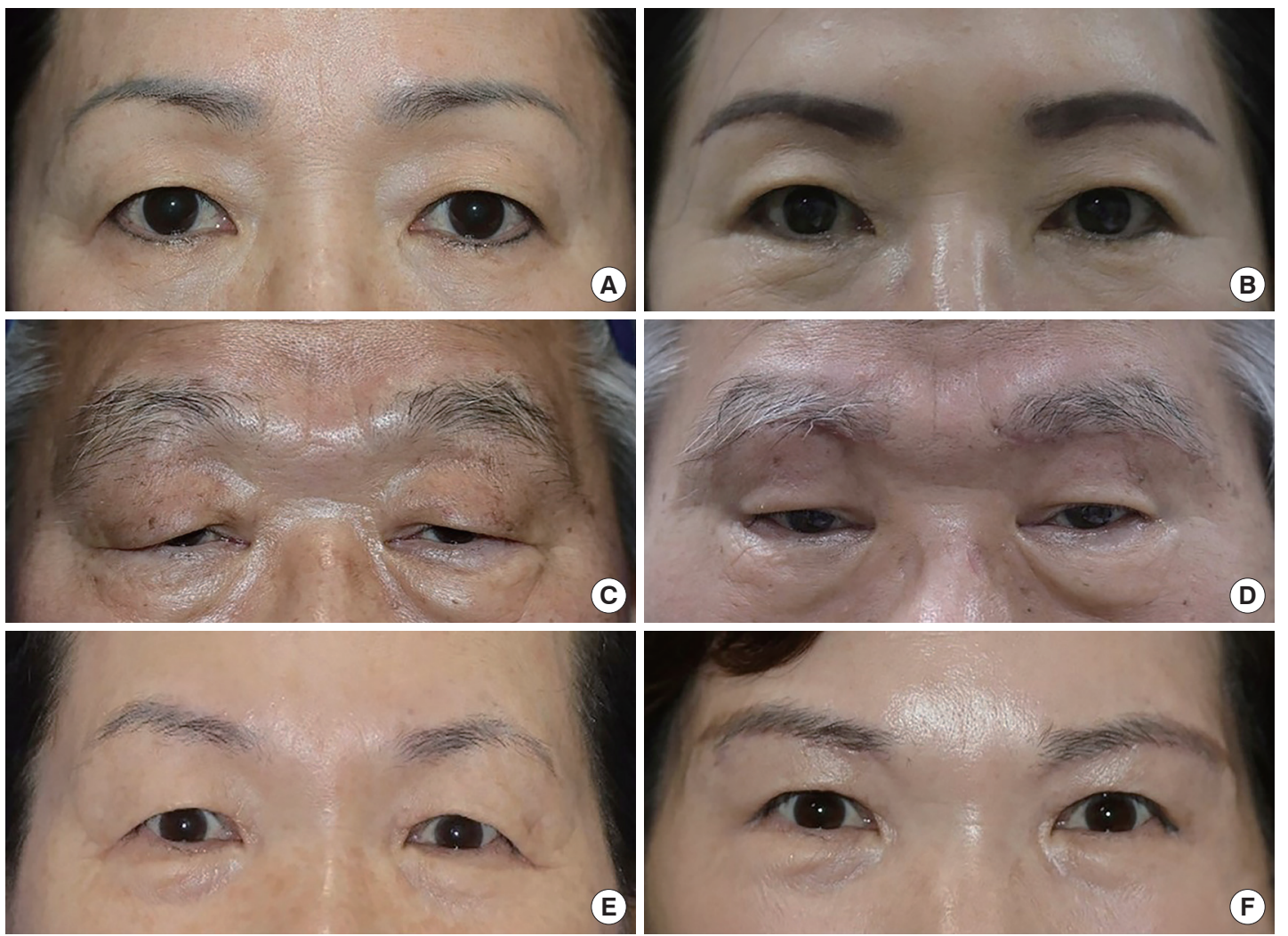

Fig. 4. Surgical case series. (A, B) A 59-year-old woman with resolution of lateral hooding and upper lid drooping. Even though makeup was applied over the surgical scar, it was already inconspicuous. (C, D) A 75-year-old male with serious visual disturbance and upper lid drooping. As he did not desire a lid crease, the primary goal for this patient was improvement of the visual field. (E, F) A 62-year-old woman achieved a more open and youthful appearance with the resolution of lateral hooding. 


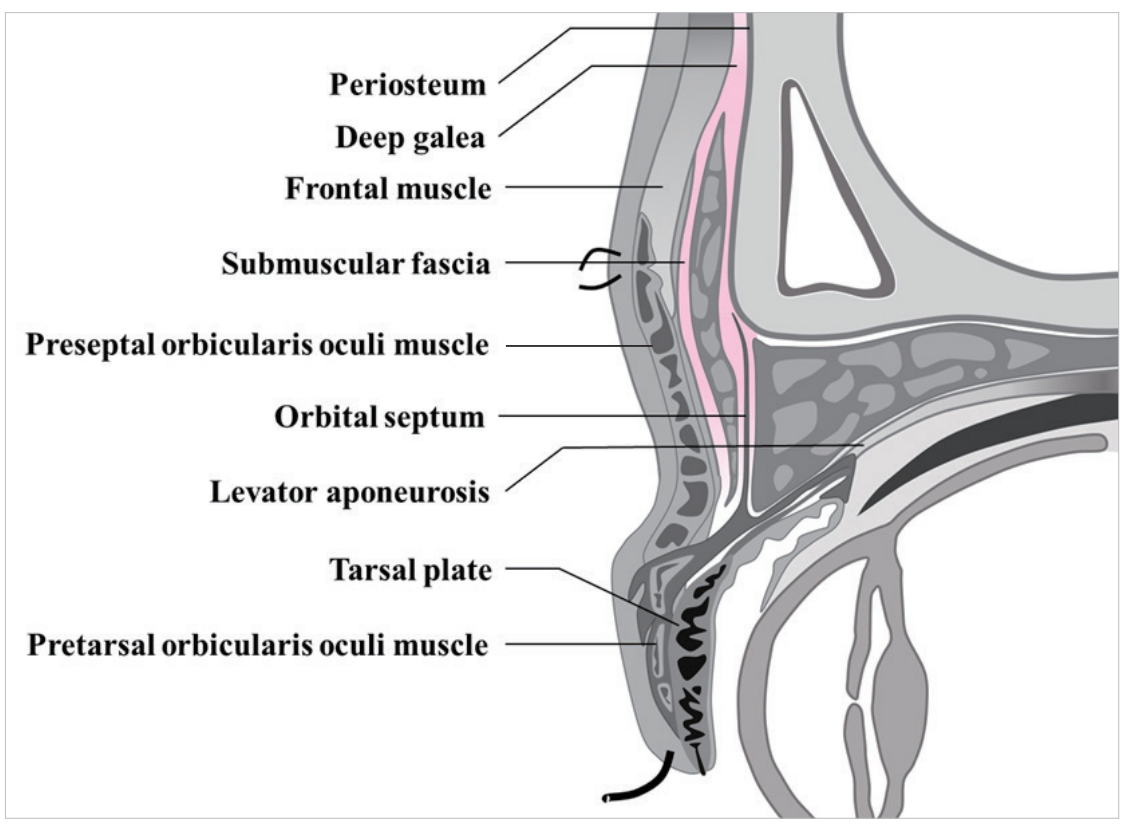

Fig. 6. The submuscular fascia, which is on the deep galea plane on lateral portion of the periorbital region, located posterior to the orbicularis oculi muscle. the patients was 60 years (range, $43-78$ years). The average followup period was 11.27 months (range, 6-25 months) (Table 1).

The preoperative mean LBD-L was 23.20 and $23.19 \mathrm{~mm}$ on the right and left, respectively. The mean LBD-L at 6 months postoperatively was 22.40 and $22.37 \mathrm{~mm}$ on the right and left, respectively. The average difference between the preoperative and postoperative LBD-L measurements was -0.800 and $-0.833 \mathrm{~mm}$ on the right and left, respectively $(\mathrm{P}=0.047$ and $\mathrm{P}=0.070)$ (Table 2$)$. The average resection width was $9.5 \mathrm{~mm}$ (range, $8-12 \mathrm{~mm}$ ).

Most patients were satisfied with the postoperative outcomes, including improvement of their visual field, a lightened feeling of the lateral lids, and a rejuvenated appearance. Reviving the eyebrow contour and providing a natural-appearing eyelid crease with inconspicuous scars yielded the most positive feedback from patients (Fig. 4).

No serious complications related to wound dehiscence, lagophthalmos, brow ptosis, sensory changes, or hypertrophic scars were reported.

\section{DISCUSSION}

As Korea becomes an aging society with access to advanced surgical techniques, older Koreans may request not only aesthetic blepharoplasty, but also improvement of their visual field.

For the past few decades, conventional blepharoplasty through a supratarsal incision to excise redundant skin and muscle has been used to manage changes associated with aging, such as dermatochalasis and baggy eyelids. However, this technique has drawbacks, including insufficient removal of lateral skin and an unnatural postoperative appearance. Moreover, classic blepharoplasty cannot ad- dress lateral hooding unless a lengthy excision is designed beyond the lateral canthus [6].

Subbrow upper blepharoplasty was first described by Parkes et al. [7] as a method to correct skin redundancy between the eyebrow and upper lid. In Asia, this technique was introduced by Sugimoto and has been widely performed in Korea and Japan [4,8]. This technique has been found to be more suitable for Asians, who have higher eyebrows than Caucasians and more pretarsal and suborbicularis fat, and are therefore vulnerable to the drawbacks of conventional upper blepharoplasty.

Several earlier studies described subbrow upper blepharoplasty using a different approach. Kim et al. [8] applied infrabrow excision blepharoplasty in Asian women, reported that the technique was useful, and presented potential indications. The supraorbital rim periosteal fixation technique was first reported by Lee and modified by Kim [5].

However, most previous studies had the limitation of analyzing the effects of subbrow upper blepharoplasty using subjective criteria, with a lack of objective information. The importance of preoperative analysis of ocular adnexal measurements has been clearly described in other studies [9]. Therefore, our study focused on obtaining information with objective values that could represent postoperative changes.

To achieve our goal of a more natural-looking contour, we hypothesized that the distance between the eyebrow and upper lid margin after surgery would be the same or minimally changed.

According to Gunter and Antrobus [10] an aesthetically pleasing brow peaks somewhere between the lateral limbus and lateral canthus. Ideally, the distance between the eyebrow and upper lid margin is the widest at the same location. Moreover, the LBD-L is 
the feature that can be most strongly affected by blepharoplasty, along with resolution of lateral hooding. Therefore, we decided to measure the LBD-L as a measure of postoperative outcomes.

Although the mean distance between the eyebrow and upper lid margin was shortened by only 0.800 and $0.833 \mathrm{~mm}$ on the right and left, respectively $(\mathrm{P}=0.047$ and $\mathrm{P}=0.070)$, the results were aesthetically more harmonious, similar to the effect of rhytidectomy in the periorbital region, as reported in another study [4]. The positive feedback we received from our patients indicated that they were satisfied with their natural and youthful appearance.

Additionally, the minimal change in LBD-L can be analyzed from two different points of view. The first perspective is the relationship between the resection width and the change of LBD-L. We would logically expect a smaller change in LBD-L if the resection width is shorter. However, since the Spearman correlation coefficient for these two variables was 0.08 and 0.185 on the right and left, respectively $(\mathrm{P}=0.673$ and $\mathrm{P}=0.328)$, this trend was not statistically significant.

Another result that needs to be emphasized is that even though our operation was performed without distinguishing between double eyelid crease and single eyelid crease, there was no statistically significant association between the eyelid crease and the change of LBD-L using the Fisher exact test $(\mathrm{P}=1.000$ and $\mathrm{P}=0.503$ on the right and left sides, respectively).

This seemingly paradoxical association between a minimal change in LBD-L and high levels of aesthetic satisfaction may reflect the fact that the actual distance is disguised by skin laxity. Although the redundant skin was resected, the upper lid skin can stretch to recover the original distance between the eyebrow and upper lid margin. This effect can be maximized in combination with fat removal to reduce the upper lid volume.

Another explanation for this association is the resolution of lateral hooding. No matter the cause, most of our patients had lateral hooding that interfered with the visual field and resulted in an aged appearance. Therefore, resolution of lateral hooding not only provided a younger appearance, but also improved the visual field without a clear change in LBD-L. This feature of subbrow upper blepharoplasty shows the potential of combining this procedure with browpexy and muscle plication or other procedures [11].

Subbrow upper blepharoplasty is widely performed in Asia using various operative techniques and criteria. The following are some modifications and clinical points to be noted regarding our subbrow upper blepharoplasty technique that were not reported in previous studies.

First, according to several studies, the anchoring depth of the inferior flap is anatomically uncertain, but is critical in reducing complications such as wound dehiscence and hypertrophic scarring [12]. To determine the appropriate anchoring depth of the inferior flap, we gently pulled the needle in a cephalic direction to confirm the elevation of the upper lid. This practical trick is used to reduce relapse of lateral hooding and to obtain a natural-appearing eyelid (Fig. 5).

The next surgical modification we used is the muscle-splitting technique at the fixation points. Instead of other blunt and invasive techniques, we made slit incisions on the OOM with small Metzenbaum scissors to reach the submuscular fascial plane. This technique may help the patient to recover faster, while not causing additional injury to the nerves.

The fixation plane in this technique-the submuscular fasciais not a well-known anatomic structure. It is posterior to the $\mathrm{OOM}$ on the medial portion and posterior to the frontalis muscle on the lateral portion (Fig. 6). It can be recognized by gently pulling on the inserted needle after placing the suture with adequate resistance and little laxity. The reason for this pulling procedure is that fixation on the periosteum can result in dimpling of the skin and displacement of the eyebrows.

In summary, subbrow upper blepharoplasty can simultaneously yield functional and cosmetic improvements. It is a flexible technique that can be modified and combined in any clinical setting. Howev$\mathrm{er}$, the main obstacle in our experience is that the indications are limited to certain populations; moreover, the technique is not recommended for severe ptosis. Further studies comparing this technique with other operative techniques are required.

Subbrow upper blepharoplasty with submuscular fascia fixation helps individuals with lateral hooding of the upper eyelid, while overcoming some drawbacks of conventional upper blepharoplasty and preventing the creation of a definite change in the periorbital contour.

In this study, we measured the lid-to-brow distance and confirmed that this procedure resulted in a minimal change, but the results were aesthetically favorable. This seemingly paradoxical finding can be explained by the characteristics of the surgical procedure, which alleviates lateral hooding, as objectively confirmed using photographs.

In conclusion, we strongly believe that among the various blepharoplasty techniques, subbrow upper blepharoplasty with submuscular fascia fixation has definite usefulness for Asians with lateral hooding. However, further research with more data is needed.

\section{NOTES}

\section{Conflict of interest}

No potential conflict of interest relevant to this article was reported.

\section{Ethical approval}

The study was approved by the Institutional Review Board of ChungAng University Hospital (IRB No. 1902-001-16245) and performed in accordance with the principles of the Declaration of Helsinki. 


\section{Patient consent}

The patients provided written informed consent for the publication and the use of their images.

\section{ORCID}

Woo Ju Kim https://orcid.org/0000-0002-7817-0443

Han Koo Kim https://orcid.org/0000-0002-2849-3973

Tae Hui Bae https://orcid.org/0000-0002-0342-1439

Woo Seob Kim

\section{REFERENCES}

1. Lee D, Law V. Subbrow blepharoplasty for upper eyelid rejuvenation in Asians. Aesthet Surg J 2009;29:284-8.

2. Kunjur J, Sabesan T, Ilankovan V. Anthropometric analysis of eyebrows and eyelids: an inter-racial study. Br J Oral Maxillofac Surg 2006;44:8993.

3. Starck WJ, Griffin JE Jr, Epker BN. Objective evaluation of the eyelids and eyebrows after blepharoplasty. J Oral Maxillofac Surg 1996;54:297302.

4. Ichinose A, Sugimoto T, Sugimoto I, et al. Extended infrabrow excision blepharoplasty for dermatochalasis in Asians. Arch Facial Plast Surg

2011;13:327-31.

5. Kim YS. Subbrow blepharoplasty using supraorbital rim periosteal fixation. Aesthetic Plast Surg 2014;38:27-31.

6. Osaki MH, Osaki TH, Osaki T. Infrabrow skin excision associated with upper blepharoplasty to address significant dermatochalasis with lateral hooding in select Asian patients. Ophthalmic Plast Reconstr Surg 2017;33:53-6.

7. Parkes ML, Kamer FM, Merrin ML. Infrabrow lift. Laryngoscope 1976; 86:1869-72.

8. Kim YS, Roh TS, Yoo WM, et al. Infrabrow excision blepharoplasty: applications and outcomes in upper blepharoplasty in Asian women. Plast Reconstr Surg 2008;122:1199-205.

9. Ing E, Safarpour A, Ing T, et al. Ocular adnexal asymmetry in models: a magazine photograph analysis. Can J Ophthalmol 2006;41:175-82.

10. Gunter JP, Antrobus SD. Aesthetic analysis of the eyebrows. Plast Reconstr Surg 1997;99:1808-16.

11. Fang YH, Liao WC, Ma H. Infraeyebrow blepharoplasty incorporated browpexy in an Asian population. Ann Plast Surg 2013;71 Suppl 1: S20-4.

12. Hwang K, Kim DJ, Hwang SH. Thickness of Korean upper eyelid skin at different levels. J Craniofac Surg 2006;17:54-6. 